\title{
Blockchain Technology and its Types-A Short Review
}

\author{
P.K. Paul ${ }^{*}$, P.S. Aithal ${ }^{2}$, Ricardo Saavedra ${ }^{3}$ and Surajit Ghosh ${ }^{4}$ \\ ${ }^{1}$ Executive Director (MCIS) and Asst. Professor (IST), Department of CIS, E Information Scientist (Offg.), Raiganj \\ University, West Bengal, India \\ ${ }^{2}$ Vice Chancellor, Srinivas University, Karnataka, India \\ ${ }^{3}$ Director \& Chair, International Program, Azteca University, Mexico \\ ${ }^{4}$ Director E Chairman, Kotibarsha Institute of Technology and Management (KITM), West Bengal, India
}

"Corresponding author: pkpaul.infotech@gmail.com

Received: 14 Oct., 2021

Revised: 13 Nov., 2021

Accepted: 05 Dec., 2021

\begin{abstract}
Blockchain Technology is the emerging component of IT and Computing and is a kind of encrypted record of data works on distributed database. This is furthermore dealt with the data related to the transaction, contract, independent record, etc. Digital ledger is important and also applicable in various platforms, not bounded in a particular place; and required in financial activities and services and hence it is worthy in healthy digital currency and transactions. Financial transaction is not associated with the third party and it keeps the data encryption and here participants no need to share personal data and thus Blockchain Technology is associated with the data breach. Insignificantly Blockchain not only reduces the data breach but also supports the multiple numbers of shared copies and it works on the same database. It helps in wage a data breach attack. Earlier it was known as Blockchain but gradually it is treated as a Technology and considered as important in Information Technology and impacting the financial sector rapidly. Blockchain Technology is required in various tangible and intangible asset management and required in tracking including recorded effectively within a network and ledger. Blockchain is therefore is a tool, a technique, as well as a procedure for sophisticated financial management. This paper is based on existing literature emphasizing Blockchain Technology with features, characteristics with a focus on its types.
\end{abstract}

Keywords: Blockchain Technology, Financial Technologies, Business Sector, Financial Engineering, IT Applications

The Blockchain is only allowed to works for the registered members having permission and this offers immediate, shared and completely transparent information. It is worthy to note that the applications of Blockchain in tracking orders, payments, accounts, and production. Here furthermore transaction details are visible to the end users from time to time. Blockchain Technology is applicable in different

How to cite this article: Paul, P.K., Aithal, P.S., Saavedra, R. and Ghosh, S. (2021). Blockchain Technology and its Types-A Short Review. IJASE., 9(02): 189-200. 
areas and sectors such as business and commerce, education and training, health and medical systems, government and administration, entertainment, banking and trade, etc., and such applications are increasing gradually[1],[9],[18]. Therefore, Blockchain Technology is a kind of fraud resistant system and holds the potential to revolutionize the financial sector transparently and advanced. Blockchain Technology is offered many benefits (such as Fig. 1) more efficiently than that of traditional business processes and its applications also growing in various sectors and places. However, it would be worthy to note that there are shortages in manpower development requirements and as a result,various educational institutions started offering training, academic, and research program in Blockchain Technologies including allied areas ${ }^{[2],[3]}$.

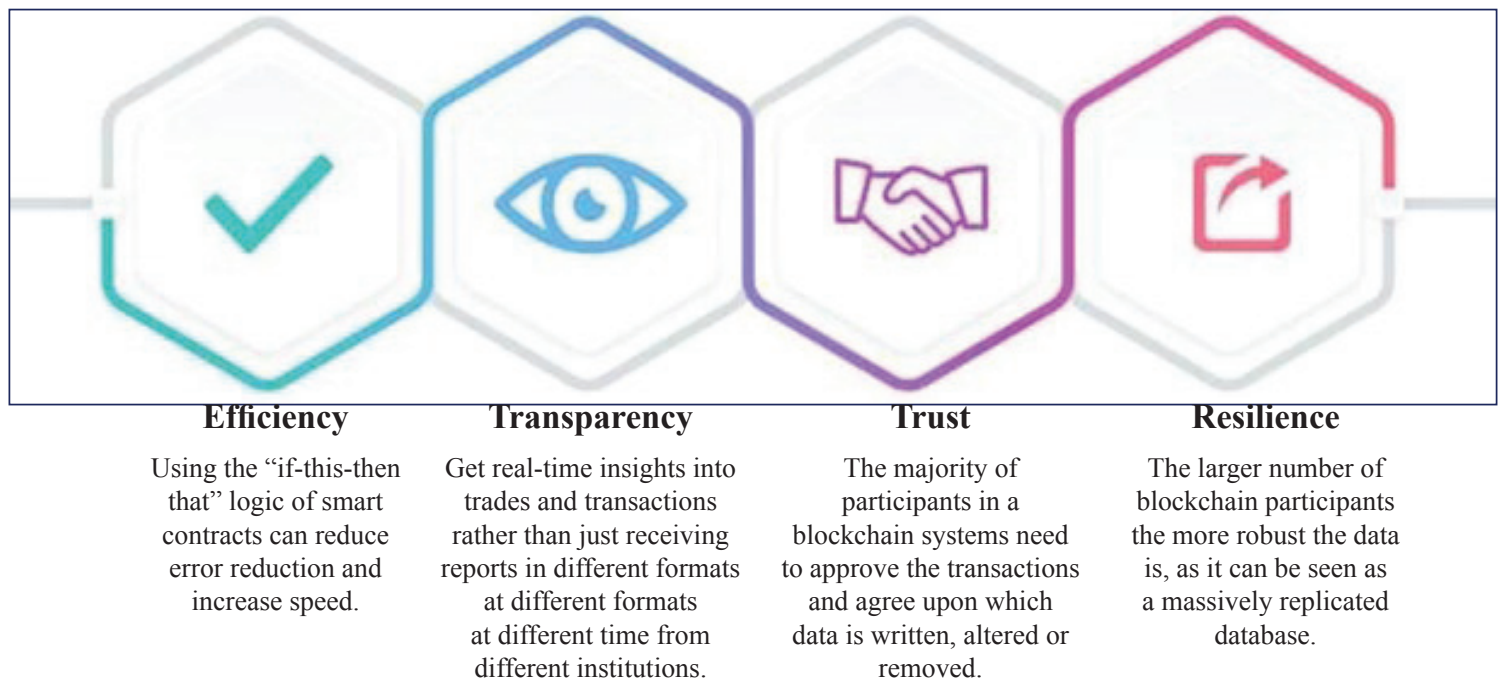

Fig. 1: Basic benefits of Blockchain Technology at a glance

\section{Objective of the Work}

This Paper entitled 'Blockchain Technology and its Types-A Short Review' is conceptual nature, and theoretical and concern about the following objectives-

To learn about the basics of Blockchain Technology with fundamental aspects including its nature.

$\square$ To know about the features and functions of Blockchain Technology including basic applications of Blockchain Technology.

$\square$ To learn the main types of blockchain with their features and functions in a concise manner.

$\square$ To know about the advantages and disadvantages of public, private, and hybrid blockchain technology.

$\square$ To learn about the basic features of the consortium blockchain in a brief and concise manner.

\section{Methodology Adopted}

This paper is theoretical in nature and various types of secondary sources and primary sources are being used. Furthermore, various secondary sources are accessed regarding Blockchain Technology including 
the features, functions, and types. Various websites of different Blockchain Technology companies have been analyzed to learn about the latest things going on in blockchain categories. In addition to previous, in regard to knowing the blockchain, various Governmental policies also studied and analyzed to prepare this work.

\section{Blockchain Technology: Basic Applications and Emergence}

Blockchain Technology is an important technology these days rather 'Blockchain' is deemed as a technology. It is not only considered as database and but also a technology with digital trust. In the year 1982 David Cham coined and proposed the term 'blockchain', and later Stuart Haber and W. Scott Stornetta also in the year 1992 do more on this technology and reported in their book ${ }^{[4],[16],[27]}$. Gradually ideas became introduced on Blockchain with the implementation of Satoshi Nakamoto after deploying the first digital currency, i.e., Bitcoin. The tools, technologies, concept, and protocols regarding Blockchain is gradually increasing. Blockchain has been gained with cryptocurrencies like bitcoin. In Blockchain, change is difficult because it is immutable, and transactions are stored in various places on the computer network. Blockchain Technology is applicable and important in different areas due to its holding of features and services such as-

Using Blockchain Medical and health data can be shared effectively.

$\square$ NFT market and sectors are applicable using proper blockchain technology.

$\square$ It is applicable in the Music royalties tracking

$\square$ It is being used in Cross-border payments with the real-time IoT operating systems

$\checkmark$ It is required in the personal identity security management and up-gradation.

$\square$ In the Anti-money laundering tracking system too Blockchain Technology is being used.

$\square$ Regarding sophisticated Supply chain and logistics monitoring and management Blockchain Technology is effectively used.

$\square$ Regarding proper, and healthy logistics monitoring Blockchain Technology are effective.

$\square$ As far as the latest Voting mechanism is concerned this technology is fruitful.

$\square$ In contemporary Advertising, marketing including Cryptocurrency exchange also blockchain is effectively used.

$\square$ Regarding real estate processing and other areas applications Blockchain Technology is worthy and demanding ${ }^{[6,[7]}$.

Therefore, Blockchain technology utilizations are gradually increasing in diverse sectors like Financial Services, Education and Training, Healthcare, Government and Administration, Travel and Hospitality, Retail, etc. ${ }^{[10],[12]}$. Here Fig. 2 illustrated about the potential advantages. 


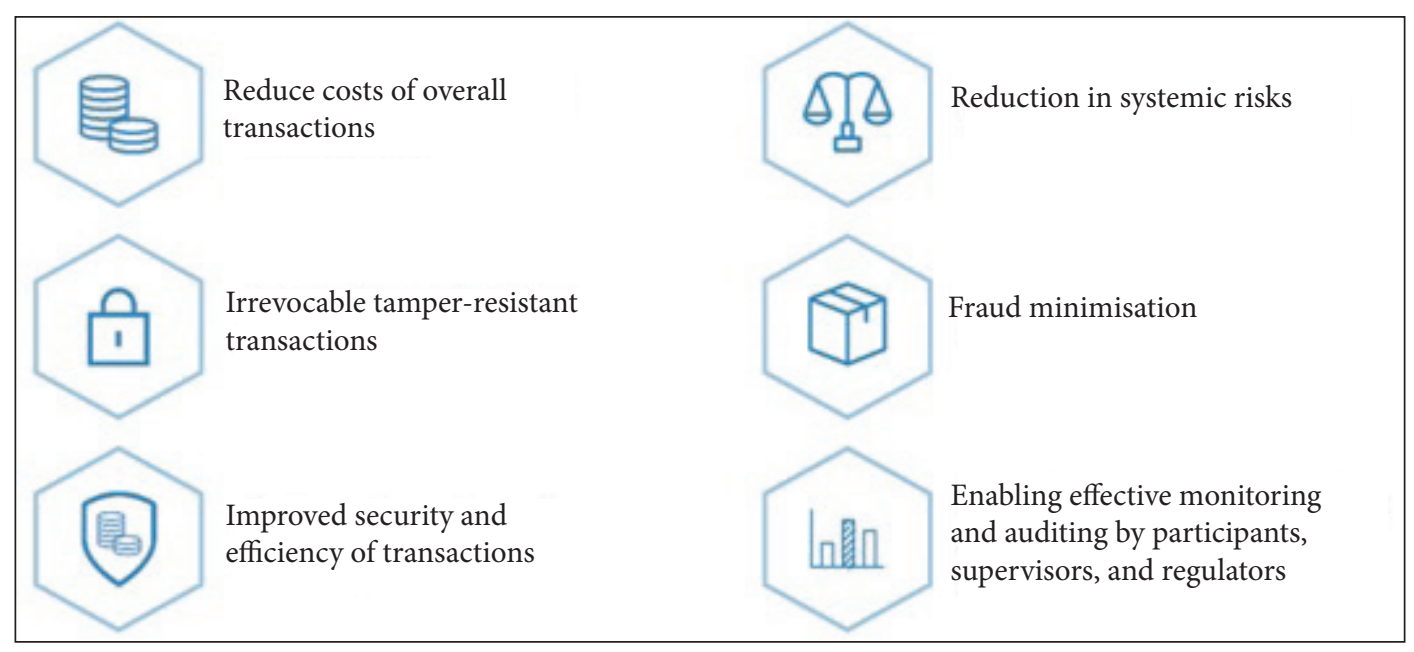

Fig. 2: Healthy and Potential advantages of blockchains

\section{Blockchain \& Types}

Blockchain technology is being used to carry and transfer the transactions or exchange of information through a secure network. Blockchain technology and distributed ledger technology is used parallel to the digital cryptocurrency to the people. Blockchain is being used for the purpose of private networking and uses too where only the restricted network users can get the authorization and access. Here network administrators are authorized to administrate the activities and any new nodes or users who wish to get permission, need to contact with the system or network administrators. Primarily there are two types of Blockchain technology viz. private Blockchain and public Blockchain. Though based on some other criteria and analysis Blockchain technology can also be noted and called as consortium blockchain technology, and hybrid blockchain technology. It is important to note that every kind of Blockchain basically consists of a cluster of nodes, and this is working on the peer-to-peer (P2P) network system. Every node in the network has a copy of the shared ledger and further that is timely updated and also being verified the transactions, initiate and receive transactions. Keeping in mind the broad nature, experts classified Blockchain Technology into following three ${ }^{[3],[21],[26]}$.

$\square$ Public Blockchain,

$\square$ Private Blockchain, and

$\square$ Hybrid Blockchain.

1. Public Blockchain is a major type of Blockchain, and that is not only open but also decentralized in nature. And in this type of Blockchain technology computer networks are basically accessible to anyone interested in transactions. Here based on validation the validated person basically receives the transaction rewards and furthermore, two kinds of Proof-of-work and Proof-of-stake models are being used.

The Public Blockchain is furthermore a non-restrictive and distributed ledger system which is doesn't seek any kind of permission, and anyone having access can be authorized one to get the data or part pf the Blockchain. This kind of Blockchain also gives authorization regarding the current and past records 
verification. Additionally, this is being used for mining and exchanging cryptocurrencies ${ }^{[11],[13],[25]}$. In this segment most common is Bitcoin and Litecoin blockchains. It is mostly secure upon following strict security rules as well as methods. However, upon non-following the security protocols it may be risky. Some of the examples of this type of Blockchain are-
$\square$ Bitcoin,
$\square$ Ethereum,
$\square$ Litecoin.

Here Fig. 3 depicted some of the features and advantages regarding Public Blockchain Systems and Technology.

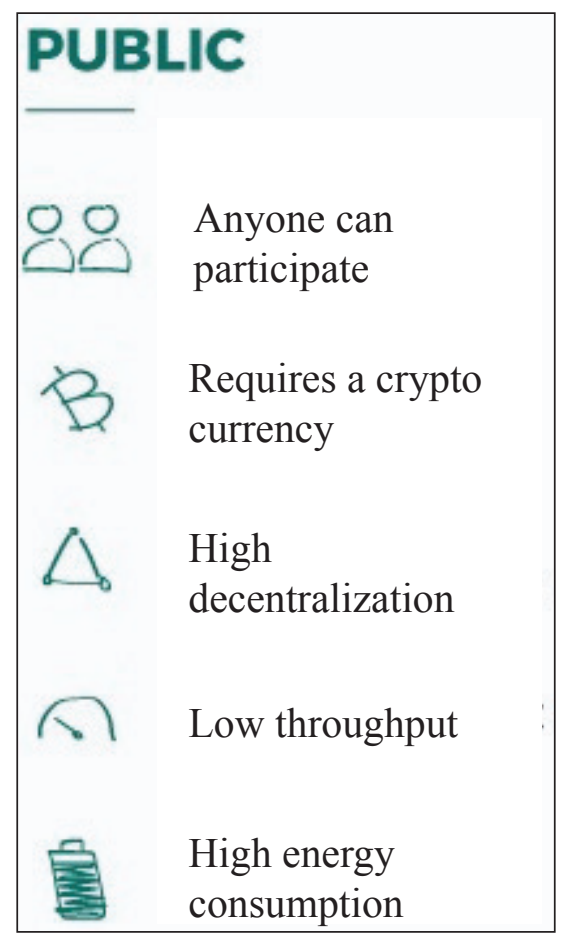

Fig. 3: Salient features and functions of the public blockchains

There are two common examples of public blockchains and these are Bitcoin and Ethereum as per the experts $^{[8],[14]}$. This type of Blockchain is concerned with the following type of features viz.

$\square$ High Security and Privacy,

$\square$ Open and Flexible Environment,

$\square$ Anonymous Nature,

$\square$ No regulations and strict Policies,

$\square$ Full Transparency and Systems.

$\square$ Distributed, etc. 
However, according to the experts, the following are being considered as important advantages and benefits of the Public Blockchain.

\section{Trustable and Faith}

Public Blockchainis trusted and here unlike private blockchains, the participants are don't need to think of authenticity. In this type of Public Blockchain, they no need of knowing other nodes, and therefore there is no fraud in the transactions. In this category nodes can contact blindly without feeling the needing to trust individual nodes ${ }^{[15],[17]}$.

\section{Secure and Safe}

In the Public Blockchain, there are opportunities in connecting with the other participants and nodes in the same public network, and this results in secure, largest, and greater communication and participation. Owing to this feature, it is difficult for the attackers to enter the systems and here every node will do the verifications and transactions as per norms. Here thoughtful cryptogenic encrypting methods are being used and therefore it is much safer than the private blockchain according to some experts.

\section{Open and Transparent}

Public Blockchain is also having the features of openness and here data is basically transparent to all the nodes and in this mechanism, one blockchain record is normally available to all the authorized nodes. Therefore, here all the nodes become open and transparent and there is an absence of fake transactions or hiding any information ${ }^{[15],[22]}$.

Though there are plenty of advantages and benefits but it is also having a different kind of disadvantages and weaknesses, and some of them are as under.

\section{Lower Transaction per Second}

In Public Blockchain System the rate of transaction per second is also very low, and this is due to having a large number of nodes and huge network. Here each node has to verify the transaction and also do proof-of-work is time consuming. Here in public systems seven (07) transactions happen per second and additionally, here Ethereum network has about a 15 TPS rate.

\section{Scalability Matters}

Similar to the previously mentioned issue on a lower transaction per second in public blockchain another issue is scalability according to the experts. The huge size basically creates the scalability in this regard and here bitcoins lightning networks are considered as important to overcome the problem according to the experts.

\section{High Energy Consumption}

The public blockchain also suffers from higher energy consumption due to the proof-of-work energy consumption. As it needs special algorithms therefore high energy consumption is considered as important in energy, environment, and financial context. 
2. Private blockchains are restricted and not open, such kind of blockchain also has features of access. This blockchain allows permission for the transaction from the support of the system administrator ${ }^{[8],[20]}$. Private blockchain solutions develop these platforms having the features of the following-

$\square$ Full of privacy,

$\square$ High efficiency,

$\square$ Faster transaction,

$\square$ Better scalability,

$\square$ Faster and speediness.

This type of blockchain is works on closed systems and networks only and these are usually useful in the organizations, enterprises from which only selected members can be joined. This type of blockchain contains proper security, authorizations, permissions as well as accessibility. According to the experts, private blockchains are deployed forvoting, regarding supply chain management, for finding and managing digital identity, regarding asset ownership, and so on. There are certain popular private blockchains like Multichain, Hyperledger projects, Corda, etc.

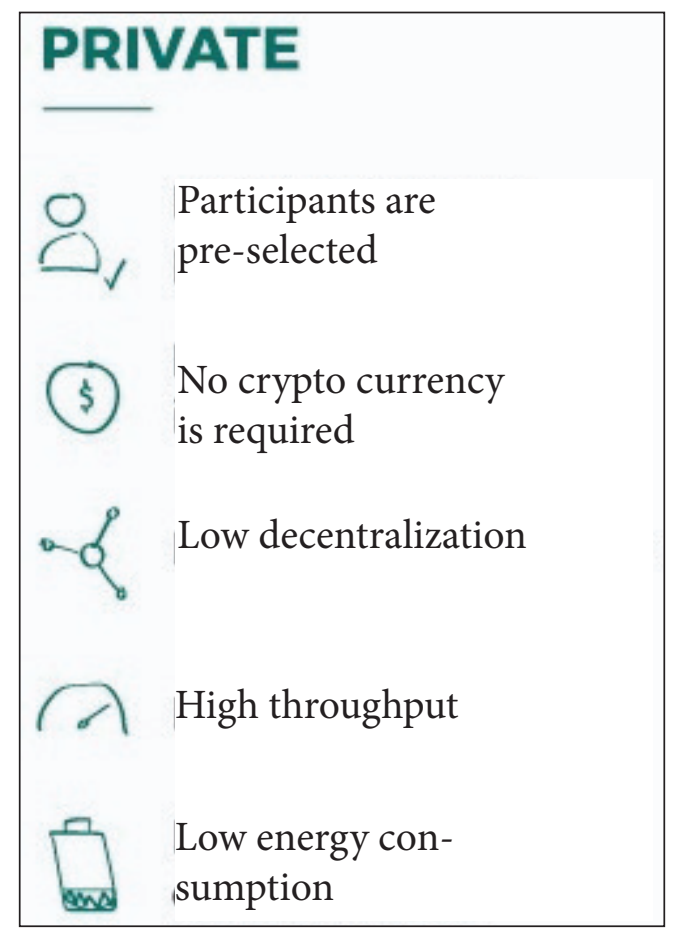

Fig. 4: Salient features and functions of the private blockchains

Private blockchains as running with the authorized nodes; therefore no one from the outside of the private network is able in accessing information and transaction related data exchanged between two nodes. Private blockchains are also held various types of advantages and disadvantages over public blockchains and among these few important are mentioned as follows. 
According to the experts, the following are being considered as important advantages and benefits of the Private Blockchain.

\section{Speed and Velocity}

Private blockchains works with greater speed than a public blockchain and therefore here higher rate of the TPC (i.e., transaction per second) can be noted. Moreover, here only a limited number of nodes can be seen therefore it comes with higher speed. Here all the nodes are having verification processability and thus here the rate of adding new transactions in a block is fast. Here about thousands or hundred thousand TPS is possible at a time ${ }^{[23],[24]}$.

Scalability

Then that of a public blockchain, a Private blockchain is faster and thus gives higher scalability. Here adding nodes to existing ones becomes easy and faster. Thus, it makes private blockchains very scalable and flexible. And here adding or removing nodes not affect as such in the existing systems.

Though there are plenty of advantages and benefits but it is also having a different kind of disadvantages and weaknesses, and some of them are as under.

\section{Requires Trust-building}

Public blockchain is a kind of open ledger and therefore it concerned with the security and legitimacy of each individual users however in Private blockchain as only limited accessed users therefore it requires trust building.

\section{Lower Security}

Private blockchain is weak when a third-party gains access to the central management system; therefore, here it is easier for a node to hack the entire private blockchain system.

3. Hybrid Blockchains is a merger of public blockchain as well as private blockchain and it is required in better control for achieving higher goals. Hybrid Blockchain deals with centralized and decentralized systems and it is not open; however, it has the features of integrity, transparency, as well as security. It has several advantages over traditional blockchains as depicted in Fig. 5.

In Hybrid Blockchains maximum customization is being considered as main benefits with private permission-based system as well as a public permission-less system. In this type of blockchain systems users are able in getting access and selected sections and rest can be recorded or keeps safe due to the benefits of the records from the ledger. Hybrid Blockchains is flexible enough so that users can join easily as private blockchain. This type of blockchain is able in enhancing the security and transparency of the blockchain network ${ }^{[16],[26]}$.

4. Consortium Blockchain is another type of semi-decentralized type of blockchain, and this type of blockchain is able in the organization of managing the blockchain network. This type of blockchain is able in doing activities even from a single organization. Here blockchain is able in exchange information or do the mining and are being used in the areas such as banks, government organizations, etc. Some of the examples of this type of consortium are Energy Web Foundation, R3, etc. 


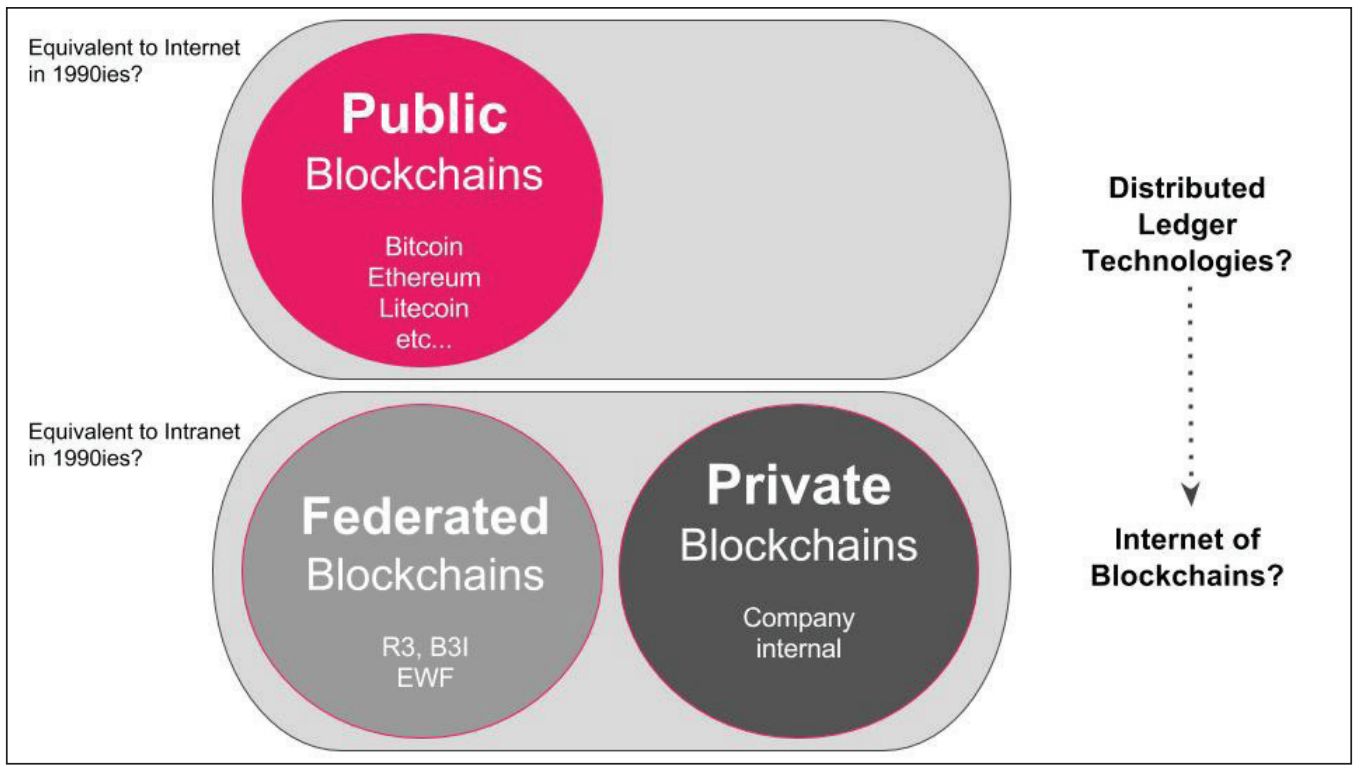

Fig. 5: Types of Blockchains and roadmaps

Therefore, in a nutshell, all the Blockchains are having their own benefits and advantages and as a whole public and private is considered as major or worthy in terms of operations (as depicted in Fig. 6) According to the experts security, scalability, and transparency are considered as worthy and main points in the Blockchains of public and private types. It is important to note that private blockchains are not trustworthy; while the public network is important in proof-of-work based.

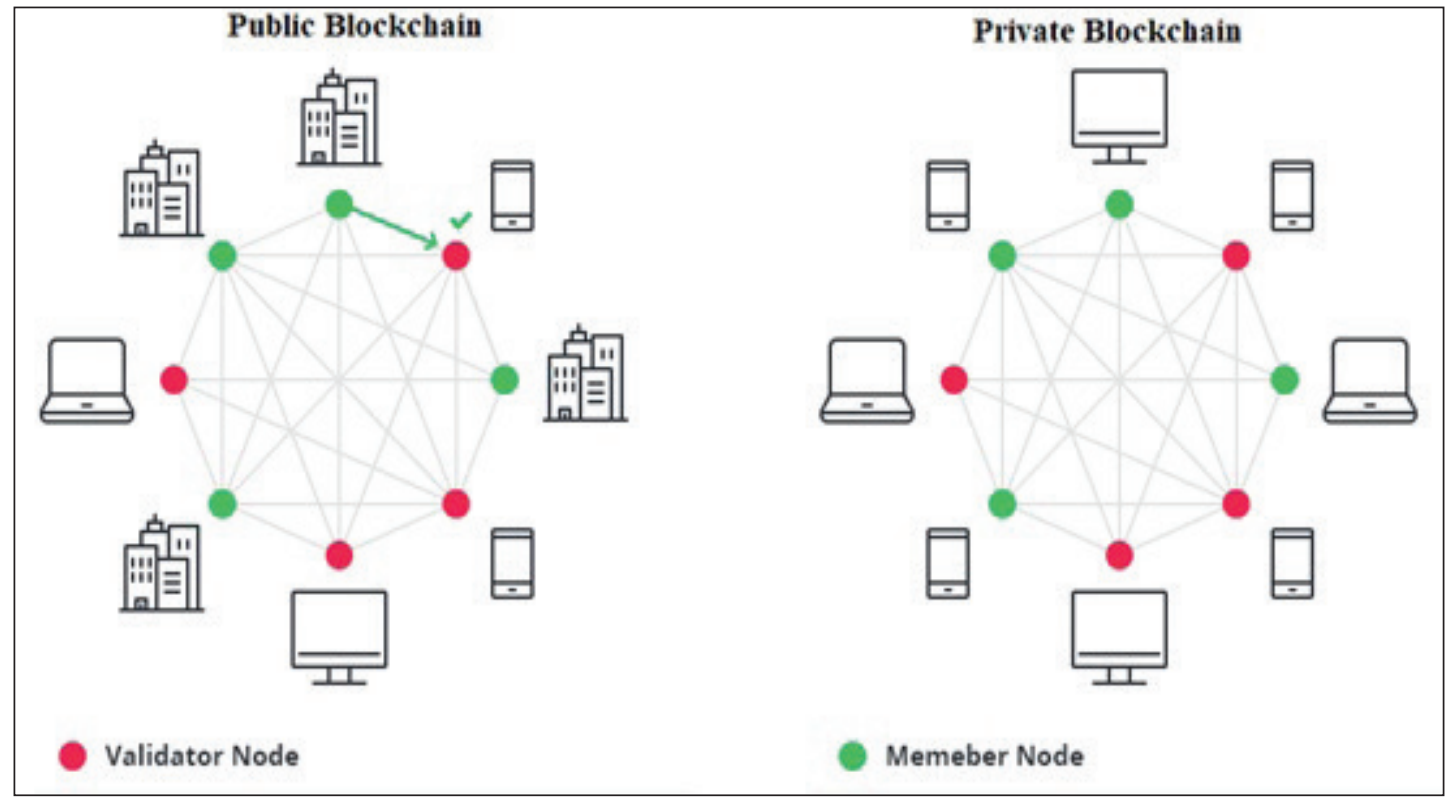

Fig. 6: Architecture wise differences in public and private blockchains 
Therefore, in a summary, it is better to know that public blockchains are worthy and useful in a different context.

\section{CONCLUSION}

Blockchain Technology is an important Information Technology component and is popular internationally and considered as worthy in peer-to-peer decentralized distributed ledger based systems. It is valuable in digital asset transparencies as in this technology third-party or intermediary or brokers are less. Here more public attention is needed to the financial market as well as organizations in a scalable manner. Many developing nations are in process of popularization of Blockchain Technology towards its worthy benefits in organizations, different organizations, institutions, businesses, and commercial ventures are engaged in blockchain related aspects. Incubation centers are dedicated in Blockchain Technology development and getting its benefits considering various challenges and issues.

\section{REFERENCES}

1. Chen, G., Xu, B., Lu, M. and Chen, N.S. 2018. Exploring blockchain technology and its potential applications for education. Smart Learning Environments, 5(1): 1-10.

2. Chattu, V.K., Nanda, A., Chattu, S.K., Kadri, S.M. and Knight, A.W. 2019. The emerging role of blockchain technology applications in routine disease surveillance systems to strengthen global health security. Big Data and Cognitive Computing, 3(2): 25-35.

3. Christ, K.L. and Helliar, C.V. 2021. Blockchain technology and modern slavery: Reducing deceptive recruitment in migrant worker populations. Journal of Business Research, 131(1): 112-120.

4. Gabison, G. 2016. Policy considerations for the blockchain technology public and private applications. SMU Sci. \& Tech. L. Rev., 19(1): 327-334.

5. Gamage, H.T.M., Weerasinghe, H.D. and Dias, N.G.J. 2020. A survey on blockchain technology concepts, applications and issues. SN Computer Science, 1(2): 1-15.

6. Gereffi, G., Wadhwa, V., Rissing, B. and Ong, R. 2008. Getting the numbers right: International engineering education in the United States, China, and India. Journal of Engineering Education, 97(1): 13-25.

7. Grover, M., Reinicke, B. and Cummings, J. 2016. How secure is education in Information Technology? A method for evaluating security education in IT. Information Systems Education Journal, 14(3): 29-37.

8. Jirgensons, M. and Kapenieks, J. 2018. Blockchain and the future of digital learning credential assessment and management. Journal of Teacher Education for Sustainability, 20(1): 145-156.

9. Kapur, D. and Mehta, P.B. 2004. Indian higher education reform: From half-baked socialism to half-baked capitalism. Center for International Development Working Paper, 103.

10. Khezr, S., Moniruzzaman, M., Yassine, A. and Benlamri, R. 2019. Blockchain technology in healthcare: A comprehensive review and directions for future research. Applied Sciences, 9(9): 1736-1745.

11. Kumar, N.M. and Mallick, P.K. 2018. Blockchain technology for security issues and challenges in IoT. Procedia Computer Science, 132(1): 1815-1823. 
12. Kursh, S.R. and Gold, N.A. 2016. Adding fintech and blockchain to your curriculum. Business Education Innovation Journal, 8(2): 6-12.

13. Lin, W., Huang, X., Fang, H., Wang, V., Hua, Y., Wang, J. ... and Yau, L. 2020. Blockchain technology in current agricultural systems: from techniques to applications. IEEE Access, 8: 143920-143937.

14. Macrinici, D., Cartofeanu, C. and Gao, S. 2018. Smart contract applications within blockchain technology: A systematic mapping study. Telematics and Informatics, 35(8): 2337-2354.

15. Milovanova, M.M., Markova, T.S., Mushrub, V., Ordynskaya, M.E. and Plaksa, J.V. 2020. Business education: training in the use of blockchain technology for business development. Revista Inclusiones, pp. 408-420.

16. Omar, I.A., Jayaraman, R., Salah, K., Yaqoob, I. and Ellahham, S. 2021. Applications of blockchain technology in clinical trials: Review and open challenges. Arabian Journal for Science and Engineering, 46(4): 3001-3015.

17. Pane, J., Verhamme, K.M., Shrum, L., Rebollo, I. and Sturkenboom, M.C. 2020. Blockchain technology applications to postmarket surveillance of medical devices. Expert Review of Medical Devices, 17(10): 1123-1132.

18. Paul, P.K., Bhuimali, A. and Chatterjee, D. 2016. Retail Informatics: Basics and emerging scenario with special reference to Design and Development of Proposed MSc-Information Science (Retail Informatics) in Indian Scenario. International Journal of Information Dissemination and Technology, 6(2): 140-144.

19. Paul, P., Aithal, P.S., Bhuimali, A. and Kumar, K. 2017. Emerging Degrees and Collaboration: The Context of Engineering Sciences in Computing \& IT-An Analysis for Enhanced Policy Formulation in India. International Journal on Recent Researches In Science, Engineering \& Technology, 5(12): 13-27.

20. Radanović, I. and Likić, R. 2018. Opportunities for use of blockchain technology in medicine. Applied Health Economics and Health Policy, 16(5): 583-590.

21. Sakhipov, A.A. and Baygozhanova, D.S. 2020. Blockchain Technology in Education. Scientific Evolution, 1(1): 36-39.

22. Siyal, A.A., Junejo, A.Z., Zawish, M., Ahmed, K., Khalil, A. and Soursou, G. 2019. Applications of blockchain technology in medicine and healthcare: Challenges and future perspectives. Cryptography, 3(1): 3 .

23. Sun, J., Yan, J. and Zhang, K.Z. 2016. Blockchain-based sharing services: What blockchain technology can contribute to smart cities. Financial Innovation, 2(1): 1-9.

24. Viriyasitavat, W., Da Xu, L., Bi, Z. and Hoonsopon, D. 2019. Blockchain technology for applications in internet of things - mapping from system design perspective. IEEE Internet of Things Journal, 6(5): 8155-8168.

25. Williams, P. 2019. Does competency-based education with blockchain signal a new mission for universities?. Journal of Higher Education Policy and Management, 41(1): 104-117. 
26. Yang, X.M., Li, X., Wu, H.Q. and Zhao, K.Y. 2017. The application model and challenges of blockchain technology in education. Modern Distance Education Research, 2, 34-45.

27. Yang, C.S. 2019. Maritime shipping digitalization: Blockchain-based technology applications, future improvements, and intention to use. Transportation Research Part E: Logistics and Transportation Review, 131: 108-117. 